\title{
A CHARACTERIZATION OF EUCLIDEAN SPACES
}

\section{R. S. PHILLIPS}

The purpose of this paper is to give an elementary proof of the fact that a Banach space in which there exist projection transformations of norm one on every two-dimensional linear subspace is a euclidean space. S. Kakutani [1] has pointed out that a modification of a proof due to Blaschke [2] will prove this theorem. F. Bohnenblust has been able to establish this theorem for the complex case by still another method. ${ }^{1}$

A Banach space is a linear, normed, complete space [3, chap. 5]. A euclidean space of dimension $\alpha$, where $\alpha$ is any cardinal number, is defined to be the Banach space of sequences $x_{\nu}$ of real numbers where $\nu$ ranges over a class of cardinal number $\alpha$, and $\sum x_{\nu}^{2}$ is finite and equal to the square of the norm [4]. We consider only spaces having at least three linearly independent elements.

P. Jordan and J. von Neumann have shown [5] that a Banach space which is euclidean in every two-dimensional linear subspace is itself a euclidean space. It is thus sufficient to show that the "unit sphere" $S$ for any three-dimensional linear subspace is an ellipsoid.

Because of the norm properties, $S$ is a convex body symmetric about the origin $o$, and contains $o$ as an interior point. Let $\gamma$ be a plane containing $o$ and let $C_{\gamma}$ be the curve of intersection of $\gamma$ and the boundary $S^{\prime}$ of $S$. The existence for each $\gamma$ of a projection operation of norm one, whose direction of projection is that of the unit vector $v_{\gamma}$, implies that the cylinder generated by lines of direction $v_{\gamma}$ tracing $C_{\gamma}$ contains $S$. Our theorem is therefore an immediate consequence of the following lemma on convex bodies (which need not be symmetric about $o$ ).

Lemma. ${ }^{2}$ If $S$ is a convex body such that for every $\gamma$ there exists a cylinder generated by $C_{\gamma}$ containing $S$, then $S$ is an ellipsoid.

We topologize the planes $\gamma$ by representing each by its direction cosines as a point on the unit sphere and using the usual topology of the unit sphere.

The proof of the lemma is divided into two parts. We first show that $v_{\gamma}$ is uniquely determined by $\gamma$, that $v_{\gamma}$ is a continuous function of $\gamma$, and that $S^{\prime}$ has a tangent plane at each of its points. It is then

${ }^{1} \mathrm{~F}$. Bohnenblust's result is not yet published.

${ }^{2} \mathrm{~W}$. Blaschke has proved a similar theorem under the assumption that there exists a tangent plane at each point of $S^{\prime}[2]$. 
easy to demonstrate that the curves of intersection for any set of parallel planes are similar. Finally we prove that such a convex body is an ellipsoid.

We state without proof the following elementary propositions about boundaries of convex bodies. $S^{\prime}$ and $C_{\gamma}$ are homeomorphs of the two-sphere and the one-sphere respectively. At each point of $C_{\gamma}$ there exist two one sided tangents. The right (or left) sided tangents at points $q$ approach the right (or left) sided tangents at $p$ as $q$ approaches $p$ from the right (or left). Furthermore $C_{\gamma}$ has a tangent at all except a denumerable set of its points. Given any one sided tangent $t$ at $p$ to $C_{\gamma}$, there exists a plane of support at $p$ containing ${ }^{3} t$. Finally given a tangent $t$ at $p$ to $C_{\gamma}$ there exist two half tangent planes to $S^{\prime}$ at $p$ each containing $t$; that is, for any $\beta \neq \gamma$ containing the line $\overline{o p}$, each one sided half tangent to $C_{\beta}$ at $p$ is contained in one of two half-planes, whose common bound is ${ }^{4} t$.

Suppose $C_{\gamma}$ has a tangent $t$ at $p$ and that $P_{1}, P_{2}$ are the half tangent planes at $p$ to $S^{\prime} . P_{1}$ and $P_{2}$ may or may not be distinct. Let $d(x)$ be the distance from $x \varepsilon S^{\prime}$ to the closest of $P_{1}$ and $P_{2}$, and let $r(x)$ be the distance from $x \varepsilon S^{\prime}$ to $p$. The convexity of $S$ then implies that $d(x) \leqq e(r) \cdot r(x)$ where $e(r) \rightarrow 0$ with $r$. For a $\beta \neq \gamma$ containing the line $\overline{o p}$, we consider any $x \varepsilon C_{\beta}$ and denote by $P_{x}$ the plane $P_{1}$ or $P_{2}$ from which $d(x)$ is measured. Then in order that the line through $x$ in the direction of $v_{\beta}$ does not pierce $S^{\prime}$, it is necessary that the component of $v_{\beta}$ perpendicular to $P_{x}$ be less than $2 \cdot e(2 r(x)) / \sin (\gamma, \beta)$ which approaches zero as $x \rightarrow p$. Hence $p+v_{\beta}$ is contained in both $P_{1}$ and $P_{2}$.

It is now easy to show that $v_{\gamma}$ is uniquely determined by $\gamma$. Suppose two such directions of projection existed and let $p$ be a point of tangency on $C_{\gamma}$ with the tangent $t$. Then there exist two distinct half tangent planes containing $t$ as their common line. The above argument shows that for any $\beta$ containing $\overline{o p}, p+v_{\beta}=t$. But this is impossible for a convex body with interior points.

\footnotetext{
${ }^{3}$ Given a one-sided tangent $t$ at $p$ to $C_{\gamma}$, there exists a plane of support at $p$ containing $t . C_{\gamma}$ is convex. Therefore $C_{\gamma}$ lies entirely on one side of any plane containing $t$. If the theorem were false, then we could find a plane containing $t$ and interior points of $S$ on both sides of the line through $t$. The convex extension of some neighborhoods about these points belongs to $S$ and intersects $\gamma$ on both sides of the line through $t$, which is impossible.

4 Given a tangent $t$ at $p$ to $C_{\gamma}$, there exist two half tangent planes at $S^{\prime}$ at $p$ each containing $t$. If the contrary were true, there would exist a $\beta$ containing the line $\overline{o p}$ such that a half tangent $t^{\prime}$ to $C_{\beta}$ at $p$ did not determine a plane of support with $t$ at $p$. The plane through $t, t^{\prime}$ therefore contains an interior point of $S$. Again the convex extension of some neighborhood of such a point and $C_{\gamma}$ belongs to $S$ and intersects $\beta$ on both sides of the line through $t^{\prime}$, which is impossible.
} 
If $v_{\gamma_{n}} \rightarrow v_{0}$ as $\gamma_{n} \rightarrow \gamma$, then $v_{0}$ defines a direction of projection for $\gamma$. If the contrary were true, there would exist $p \varepsilon C_{\gamma}$ and a real number $\lambda$ such that $q=p+\lambda v_{0}$ is an interior point of $S$. Let $\beta$ be the plane determined by $o, p, q$ and $p_{n}$ the point of $C_{\gamma_{n}} \cdot C_{\beta}$ closest to $p$. Then, as $n \rightarrow \infty, p_{n} \rightarrow p$ and the points not interior to $S, p_{n}+\lambda v_{\gamma_{n}} \rightarrow q$, which is impossible. By the uniqueness, $v_{0}=v_{\gamma}$. Since the $v_{\gamma}$ form a compact set, it follows that $v_{\gamma}$ is a continuous function of $\gamma$.

We next show that $S^{\prime}$ has a tangent plane at each of its points. Let $p \varepsilon S^{\prime}, \gamma$ contain $\overline{o p}$, and let $t$ be, say, the right sided tangent at $p$ to $C_{\gamma}$. Then there exist points $p_{n} \varepsilon C_{\gamma}$ which approach $p$ from the right and which have tangents $t_{n}$ to $C_{\gamma}$. Let us choose a subsequence for which a set of half tangent planes $P_{n^{\prime}}$ converge. For convenience we renumber this subsequence $1,2, \cdots, n, \cdots$. Suppose $P_{n} \rightarrow P$. Then $P$ contains $t$. Finally for any $\beta \neq \gamma$ containing $\overrightarrow{o p}$, let us choose a sequence $\beta_{n}$ containing $\overline{o p}_{n}$ such that $\beta_{n} \rightarrow \beta$. As above, $p_{n}+v_{\beta_{n}}$ lies in $P_{n}$ and since $v_{\beta_{n}} \rightarrow v_{\beta}, p+v_{\beta}$ lies in $P$. As $v_{\beta}$ does not lie in $\beta$, it follows that the $v_{\beta}$ for $\beta$ containing $\overline{o p}$ determine $P$. But $t$ was an arbitrary one-sided tangent at $p$. Hence $P$ contains all one sided tangents to curves $C_{\beta}(\beta$ containing $\overline{o p})$ and is therefore the tangent plane to $S^{\prime}$ at $p$.

We now define any directed line through $o$ to be the $z$ axis. The $x-y$ plane is then the plane containing $o$ which is parallel to the tangent plane to $S^{\prime}$ at the intersection $p$ of $S^{\prime}$ and the $z$ axis. In a system of cylindrical coordinates, let $\gamma_{\theta}$ be the plane $\theta=$ const. Then $v_{\gamma_{\theta}}$ lies in the $x-y$ plane. The curve of intersection $C_{z}$ of $S^{\prime}$ with the plane $z=$ const. is defined by the differential equation

$$
d r / d \theta=r F(\theta), \quad r(0)=f(z)
$$

where $F(\theta)$ is a continuous function independent of $z . S^{\prime}$ is therefore expressible in the form $r=f(z) \cdot g(\theta)$. Clearly the $C_{\gamma_{\theta}}$ differ only by a linear transformation.

We next prove that $C_{\gamma}$ is an ellipse. ${ }^{5}$ For this we need to know that there exists a linear orientation-preserving transformation sending $C_{\gamma_{0}}$ into itself and $p$ into any other point $q$ of $C_{\gamma_{0}}$.

Let $r$ be the point of tangency of a plane parallel to the $x$-z plane having a positive $y$ component. Suppose $\gamma_{1}$ is the plane defined by $p, o, r$. We have shown that $C_{\gamma_{0}}$ goes into $C_{\gamma_{1}}$ by a linear transformation which leaves invariant points of the $z$ axis. We can repeat the above construction about the line $\overline{o r}$. Hence if $\gamma_{2}$ is the plane defined

5 The remainder of the proof is similar to an argument used by Garrett Birkhoff, Duke Mathematical Journal, vol. 1 (1935), pp. 169-172, Theorem 1. 
by $q, o, r$, then $C_{\gamma_{1}}$ goes into $C_{\gamma_{2}}$ and $p$ goes in to $q$ by a linear transformation leaving. the $x-z$ plane and points of $\overline{o r}$ invariant. Repeating the above construction about the line $\overline{o q}, C_{\gamma_{2}}$ goes into $C_{\gamma_{0}}$ by a linear transformation which leaves points of $\overline{o q}$ invariant. The product of these transformations is the desired linear transformation.

The set $C_{\gamma_{0}}$ is compact and bounded away from $o$. Therefore the group of all orientation-preserving linear transformations of $C_{\gamma_{0}}$ into itself is bounded and hence equivalent, after a linear transformation, to a subgroup $G$ of the orthogonal group [6, p. 465, Theorem 19]. Since $G$ is transitive on lines through $o, G$ must be the entire orthogonal group. The set of points invariant under $G$ is the circle. Therefore a suitable linear transformation sends $C_{\gamma_{0}}$ into the circle. It follows that all $C_{\gamma}$ through $p$ are ellipses. $p$ was chosen arbitrarily. All $C_{\gamma}$ are therefore ellipses. If we now take a particular $C_{\gamma}$ and choose its major axis to be the $z$ axis of our construction, $S^{\prime}$ will be generated by this ellipse tracing an ellipse in the $x-y$ plane and rotating about the $z$ axis. $S$ is therefore an ellipsoid.

\section{REFERENCES}

1. S. Kakutani, Some characterizations of Euclidean space, Japanese Journal of Mathematics, vol. 16 (1939), pp. 93-97.

2. W. Blaschke, Kreis und Kugel, Leipzig, 1916, pp. 157-159.

3. S. Banach, Théorie des Opérations Linéaires, Warsaw, 1932.

4. H. Löwig, Acta Szeged, vol. 7 (1934), pp. 1-33.

5. P. Jordan and J. von Neumann, On inner products in linear metric spaces, Annals of Mathematics, (2), vol. 36 (1935), pp. 719-723.

6. J. von Neumann, Almost periodic functions in a group I, Transactions of this Society, vol. 36 (1934), pp. 445-492.

The Institute for Advanced Study 